\title{
A new solvothermal approach to obtain nanoparticles in the $\mathrm{Cu}_{3} \mathrm{SnS}_{4}-\mathrm{Cu}_{2} \mathrm{FeSnS}_{4}$ join
}

\author{
Andrea GIACCHERINI ${ }^{1}$, Andrea GRIESI ${ }^{2}$, Giordano MONTEGROSSI ${ }^{3}$, Maurizio ROMANELLI ${ }^{4}$, \\ Giovanni O. LEPORE ${ }^{5}$, Alessandro LAVACCHI', Georg AMTHAUER ${ }^{7}$, Günther REDHAMMER ${ }^{7}$, \\ Gerold TIPPELT'7 , Stefano MARTINUZZI ${ }^{8}$, Giuseppe CUCINOTTA ${ }^{8}$, Matteo MANNINI ${ }^{8}$, \\ Andrea CANESCHI ${ }^{1}$, Francesco DI BENEDETTO ${ }^{*}$
}

\author{
${ }^{1}$ Dept. of Industrial Engineering, University of Florence, via di Santa Marta 3, 50100 Firenze, Italy \\ ${ }^{2}$ Center of Nanotechnology@NEST, Istituto Italiano di Tecnologia, Piazza San Silvestro 12, 56100 Pisa, Italy \\ ${ }^{3}$ Institute of Geosciences and Earth Resources, CNR, via G. La Pira, 4, 50124 Firenze, Italy \\ ${ }^{4}$ Dept. of Earth Sciences, University of Florence, via G. La Pira 4, 50124 Firenze, Italy; francesco.dibenedetto@unifi.it \\ ${ }_{5}^{5}$ Operative Group in Grenoble, Istituto di Officina dei Materiali, CNR, clo European Synchrotron Radiation Facility (ESRF), \\ avenue des Martyrs, 71, 38100 Grenoble, France \\ ${ }^{6}$ Institute for the Chemistry of OrganoMetallic Compounds, CNR, via Madonna del Piano 10, 50019 Sesto Fiorentino, Italy \\ ${ }^{7}$ Dept. of Chemistry and Physics of Materials, University of Salzburg, Jakob-Haringer-Straße 2, 5020 Salzburg, Austria \\ ${ }^{8}$ Dept. of Chemistry, University of Florence, via della lastruccia 3, 50019 Sesto Fiorentino, Italy \\ ${ }^{*}$ Corresponding author
}

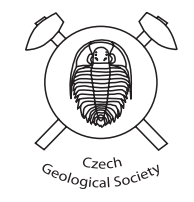

\begin{abstract}
In the field of the renewables, a large effort has been devoted in the last years to obtain conventional and new materials for solar energy conversion by using methods which couple a good efficiency and scalability with energetic and environmental concerns. This research has included the so-called kesterites, materials considered interesting for the thin-film solar cell technology, consisting of relatively abundant and harmless elements: $\mathrm{Cu}_{3-x-y} \mathrm{Fe}_{x} \mathrm{Zn}_{\mathrm{y}} \mathrm{Sn}(\mathrm{S}, \mathrm{Se})_{4}$.

In this study, we undertook the synthesis of members of the kuramite-stannite $\left(\mathrm{Cu}_{3} \mathrm{SnS}_{4}-\mathrm{Cu}_{2} \mathrm{FeSnS}_{4}\right)$ join by means of a two-step solvothermal approach, able to provide nanocrystalline products in an easy, low-temperature, and fast way. The sample with the highest Fe concentration was characterised by means of a multi-analytical approach, aimed to assess not only its final structural, chemical and micromorphological features, but also the redox speciation of the two transition metal cations, i.e. $\mathrm{Cu}$ and $\mathrm{Fe}$, in relation to the overall charge balance. Namely, Electron Paramagnetic Resonance (EPR), Mössbauer and X-ray Absorption Spectroscopy (XAS) and SQUID magnetometry were involved.

The main results point out an excellent control of the structural features, and an intermediate Fe content in the sample, leading to the following formula unit: $\mathrm{Cu}_{2.2} \mathrm{Fe}_{0.48} \mathrm{Sn}_{1.2} \mathrm{~S}_{4}$. The overall findings of the multi-analytical characterization imply a complex redox balance, where inferring the site occupancy is not trivial; the charge balance, in fact, can only be achieved taking into account the presence of both Fe(III) and vacancies. Moreover, Fe is distributed over two different crystallographic sites.
\end{abstract}

Keywords: p-type semiconductors, stannite, solvothermal synthesis, crystal chemistry, spectroscopy, magnetism Received: 31 October 2019; accepted: 17 March 2020; handling editor: F. Laufek

The online version of this article (doi: 10.3190/jgeosci.300) contains supplementary electronic material

\section{Introduction}

The large efforts paid in recent years to develop new materials for solar energy conversion and new methods to produce them have allowed to set two important conclusions. First, among renewables, solar energy conversion will never be limited in its exploitation by limitations in the energy supply; the photovoltaic devices, in fact, pay back the invested energy in their realization in few years (Alsema 2012). Second, the only up-to-date hypothesized relevant critical factor is the limitation in supplying certain strategic elements (see e.g. the case of the technology for wind energy conversion, Pavel et al. 2017).
Accounting for this general framework, a number of studies (see e.g. Cui et al. 2012; Zhong et al. 2015; Zhou et al. 2016; Hou et al. 2017; Nefzi et al. 2018; Oueslati et al. 2018; Alanazi et al. 2019; Hussein and Yazdani 2019; Mukurala et al. 2019; Nilange et al. 2019; Sanad et al. 2019; Zaman et al. 2019 a, b, c, and references therein) has been devoted to establish "green" routes to synthesize strategic photovoltaic materials (i.e. routes involving moderate thermal cycles, and limited number of chemical species involved in the synthetic pathway). An effort was also made to widen the palette of chemical compositions able to convey a desired semiconducting property. This research has mainly focused on the materials for thin-film photovoltaic devices. 
Di Benedetto et al. (2011) set the conditions for a simple, scalable, and very clean procedure to synthetize kuramite, $\mathrm{Cu}_{3} \mathrm{SnS}_{4}$, a phase belonging to the group of the so-called "kesterites", $\mathrm{Cu}_{2} \mathrm{ZnSn}(\mathrm{S}, \mathrm{Se})_{4}$. These materials are considered very promising for application in solar energy conversion (Giraldo et al. 2019). This approach couples a mild temperature treatment and the complete absence of surfactants and/or modifiers during the run. Moreover, the proposed synthesis yields products in the form of nanoparticles, a size interesting for the preparation of ink intermediates (Riha et al. 2009). The synthesis of quaternary sulfides using the same approach highlighted some difficulties. Among them, the limitation in controlling the final composition of the products, as well as the scarce control on the valence states, and site occupancies of the transition metals involved in the process. These difficulties were introduced in a study on solvothermal products in the kuramite $\left(\mathrm{Cu}_{3} \mathrm{SnS}_{4}\right)$-kesterite $\left(\mathrm{Cu}_{2} \mathrm{ZnSnS}_{4}\right)$ pseudobinary join (Giaccherini et al. 2019a). In the present study, we want to extend our investigation to stannite, $\mathrm{Cu}_{2} \mathrm{FeSnS}_{4}$, the Fe end-member of the kuramite-stannite-kesterite pseudoternary field, and to develop some general considerations on the solvothermal synthesis of complex sulfides.

Kuramite and stannite have been described to crystallize in the same structural model. Their tetragonal unit cell (kuramite: $a=5.445 \AA ; c=10.75 \AA \AA$; JCPDS

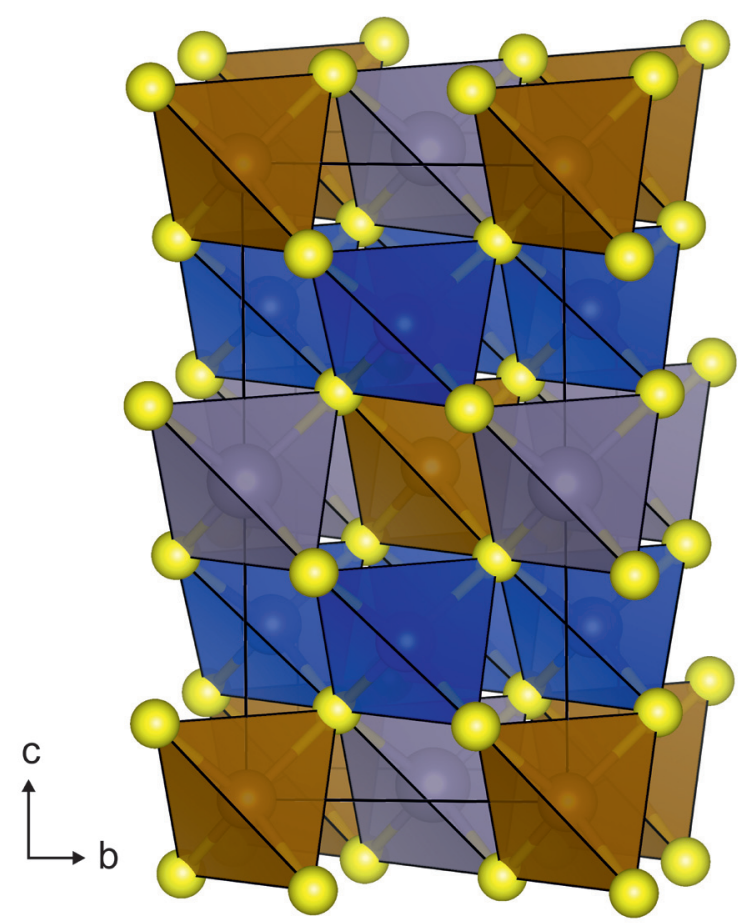

Fig. 1 The stannite tetragonal structural model. The $\mathrm{Cu}, \mathrm{Fe}$ and $\mathrm{Sn}$ tetrahedra are in blue, bronze and violet, respectively. Yellow spheres are sulfur atoms.
33-0501: Kovalenker 1981; stannite: $a=5.4495 \AA$; $c=10.726 \AA$; JCPDS 44-1476: Bonazzi et al. 2003) is in fact a 2 a supercell of a sphalerite-type subunit. In the sphalerite structure, cations and sulfur anions are located in two different cubic face-centered sublattices shifted by $(1 / 4,1 / 4,1 / 4)$. From this structure, we can derive the tetragonal model for kuramite and stannite by ordering the cationic sites along the $\mathrm{c}$ axis, giving rise to the $\overline{\overline{4}} 2 \mathrm{~m}$ (Space Group 121) structural model, where $\mathrm{Sn}^{4+}$ and $\mathrm{Cu}^{+}$are hosted in the $2 b$ and $4 d$ sites, respectively. Differently, $\mathrm{Fe}^{2+}$ in stannite and $\mathrm{Cu}^{2+}$ in kuramite are hosted in the $2 a$ site (Fig. 1).

\section{Experimental procedures}

\subsection{Synthesis and phase composition}

All syntheses of this study were performed using the reagent-grade chemicals: $\mathrm{CuCl}_{2} \cdot 2 \mathrm{H}_{2} \mathrm{O}$ (Merck), $\mathrm{FeCl}_{2}$ (Alfa Aesar), $\mathrm{SnCl}_{2} \cdot 2 \mathrm{H}_{2} \mathrm{O}$ (Riedel De Haën AG), thiourea (TU, Merck), ethylene glycol (EG, 99\%, Alfa Aesar) and milliQ water. They were carried out by a two-steps method, recently adapted for the synthesis of kesterite type quaternary sulfides (Giaccherini et al. 2019b). In the first step, the chloride of the metal-TU complexes, i.e. $\left[\mathrm{Cu}(\mathrm{TU})_{3}\right]$ $\mathrm{Cl}_{2},\left[\mathrm{Fe}(\mathrm{TU})_{4}\right] \mathrm{Cl}_{2}$ and $[\mathrm{Sn}(\mathrm{TU})] \mathrm{Cl}_{2}$, were obtained by precipitation at room temperature after having homogenized the reactants, mixed in opportune amounts (Gusain et al. 2015; Giaccherini et al. 2019b). In the second step, 0.8, 0.4 and $0.4 \mathrm{mmol}$ of $\left[\mathrm{Cu}(\mathrm{TU})_{3}\right] \mathrm{Cl}_{2},\left[\mathrm{Fe}(\mathrm{TU})_{4}\right] \mathrm{Cl}_{2}$ and $[\mathrm{Sn}(\mathrm{TU})] \mathrm{Cl}_{2}$, respectively, were dissolved in $50 \mathrm{ml}$ of $\mathrm{EG}$, and put in a two-necks flask. The solution was heated under reflux, for two hours, then let spontaneously cool down to room temperature. All operations were carried out at room pressure. Finally, the products were obtained through centrifugation, and washed several times in $\mathrm{EtOH}$ to remove any impurity of unreacted moieties. Numerous syntheses were performed to achieve a target composition of $\mathrm{Cu}_{2} \mathrm{FeSnS}_{4}$ (analogue to that of natural stannite).

The phase composition analysis of the synthetic products was carried out through powder X-ray diffraction (PXRD), using a PANalytical X'PERT PRO powder diffractometer (at the Dept. of Chemistry and Physics of Materials of the Paris-Lodron Universität in Salzburg) and employing Ni-filtered $\mathrm{Cu} K_{\alpha}(1.54187 \AA)$ radiation. The PXRD patterns were registered with a PIXcel detector on a rotating sample prepared on a silicon wafer (zero-background sample holder) in the $2 \theta$ range $5-100^{\circ}$, applying a step size of $0.026^{\circ} 2 \theta$ and a step counting time of $0.27 \mathrm{~s}$.

Refinement of the powder X-ray pattern has been carried through the GSASII software (Toby and Dreele 2013). The calibration of the instrumental broadening and of the take-off angle offset has been carried out through the $\mathrm{LaB}_{6}$ standard (NIST-SRM 660c). Two structural 
models in different space groups have been tested by means of Rietveld refinement of PXRD data. Chemical composition of the sample was attributed on the findings of the other chemical and element-specific techniques. The first one is the conventional tetragonal $(I \overline{4} 2 \mathrm{~m}-\mathrm{SG}$ 121) model for stannite structure (Bernardini et al. 1990; Bonazzi et al. 2003). With reference to the average chemical composition (discussed later on), $\mathrm{Fe}$ and an excess of Sn have been located at the $2 a$ site, Sn at the $2 b$ site, and $\mathrm{Cu}$ at the $4 d$ site. The complete disordering of the cationic sites $(4 \mathrm{~d}, 2 \mathrm{a}$ and $2 \mathrm{~b}$ ) has been considered to realize a symmetry increase to $F \overline{4} 3 \mathrm{~m}$ (SG 216) (Di Benedetto et al. 2011; Giaccherini et al. 2019b). This second model was also tested. No attempts to refine the site composition were made.

Any background signal was fitted with a Chebyshev polynomic curve and stripped from the pattern. After background removal, the refinement process has been carried out by refining scale factor, lattice constants, strain and unit cell parameters while keeping constant every other parameters. A slight improvement of the refinement has been obtained through the fit of one isotropic Debye-Waller factor for all the cationic sites and one for the sulfur site.

\subsection{Scanning-electron microscopy (SEM)}

Particle morphology was evaluated by means of the Field-Emission Scanning-Electron Microscopy (FESEM), carried out at the Centro di Microscopie Elettroniche "Laura Bonzi" (Ce.M.E.) of the Italian National Research Council (CNR), using a Tescan GAIA 3 FIB/SEM. Products were investigated at different magnifications, ranging from $20,000 \times$ up to $275,000 \times$. The powder was mounted on standard aluminum sample holders with adhesive graphite tabs. Samples were not coated to avoid possible modification of the particle surfaces, accounting for the semiconducting properties of the products.

Chemical composition of the sample was provided by an Oxford Instruments X-MAX $50 \mathrm{~mm}^{2}$ SDD EnergyDispersive X-ray spectrometer (EDX) coupled to a Zeiss Ultra Plus Field-Emission Scanning Electron Microscope (FE-SEM) at the Dept. of Chemistry and Physics of Materials of the Paris-Lodron Universität in Salzburg. Samples were mounted on standard Al-sample holders with adhesive conducting graphite tapes. Analysis was done at $20 \mathrm{kV}$ with a working distance of $9 \mathrm{~mm}$, corresponding to the best efficiency of the EDX detector.

\subsection{Electron Paramagnetic Resonance (EPR) and SQUID magnetometry}

Conventional EPR spectroscopy measurements were performed on the as-synthesized powders without further manipulation. Powders were inserted in amorphous silica tubes using a Teflon bag. Data were collected at room temperature using a Bruker ER 200D-SRC spectrometer operating at X-band $(9.5 \mathrm{GHz})$ interfaced with DS/EPR software to a PC for data acquisition and handling. Operating conditions were $0.05 \mathrm{mT}$ modulation amplitude and $100 \mathrm{kHz}$ modulation frequency. Scan speed was set to $2.5 \mathrm{mT} / \mathrm{s}$, and the investigated range was $0-1 \mathrm{~T}$.

Magnetic investigations were carried out on the assynthesized products, inserting loose powders in a Teflon bag. Magnetization measurements were performed in the 1.6-300 K temperature range using a Superconducting Quantum Interference Device (SQUID) magnetometer (MPMS Quantum Design) in the zero field-cooled (ZFC) and field-cooled (FC) modes at applied fields of 0.5 and 0.005 T. Corrections for diamagnetism, estimated from Pascal's constants (Bain and Berry 2008), were applied.

\subsection{X-ray Absorption Spectroscopy (XAS)}

$\mathrm{X}$-ray Absorption Spectroscopy measurements at the $\mathrm{K}$-edge of $\mathrm{Fe}$ were performed at the LISA beamline (BM-08) (d'Acapito et al. 2019; Puri et al. 2019) of the European Synchrotron Radiation Facility (ESRF, Grenoble - France). Samples were measured using a pair of $\mathrm{Si}$ [111] flat crystals; Si-coated focusing mirrors $\left(E_{\text {cutoff }} \approx 16\right.$ $\mathrm{KeV}$ ) were used for harmonic rejection. Measurements were performed in the transmission mode. Samples were mixed with $12.5 \mathrm{mg}$ cellulose, an amount of material sufficient to keep the total absorption $(\mu) \leq 1.5$ above the $\mathrm{Fe}$ edge, and pressed in pellets. The energy sampling interval was $0.1 \mathrm{eV}$ in the pre-edge region and $0.5 \mathrm{eV}$ in the edge region; spectra were acquired with a fixed $k$ step of 0.05 $\AA^{-1}$ up to a maximum $k$ value of $13 \AA^{-1}$. Samples were measured at room temperature. The ATHENA software (Ravel and Newville 2005) was used to average multiple spectra. Standard procedures (Lee et al. 1981) were followed to extract the structural Extended X-Ray Absorption Fine Structure (EXAFS) signal $(k \bullet \chi(k))$ : pre-edge background removal, spline modelling of bare atomic background, edge step normalization, and energy calibration. Model atomic clusters centered on the absorber atom were obtained by ATOMS (Ravel 2001), using atomic coordinates taken from Bonazzi et al. (2003); theoretical amplitude and phase functions were generated using the FEFF8 code (Ankudinov et al. 1998). The EXAFS spectra were fitted through the ARTEMIS software (Ravel and Newville 2005) in the Fourier-Transform (FT) space.

\subsection{Mössbauer spectroscopy}

${ }^{57} \mathrm{Fe}$ Mössbauer spectra were recorded using a multichannel analyzer (1024 channels) operating in conjunction with an electromechanical drive system with symmet- 

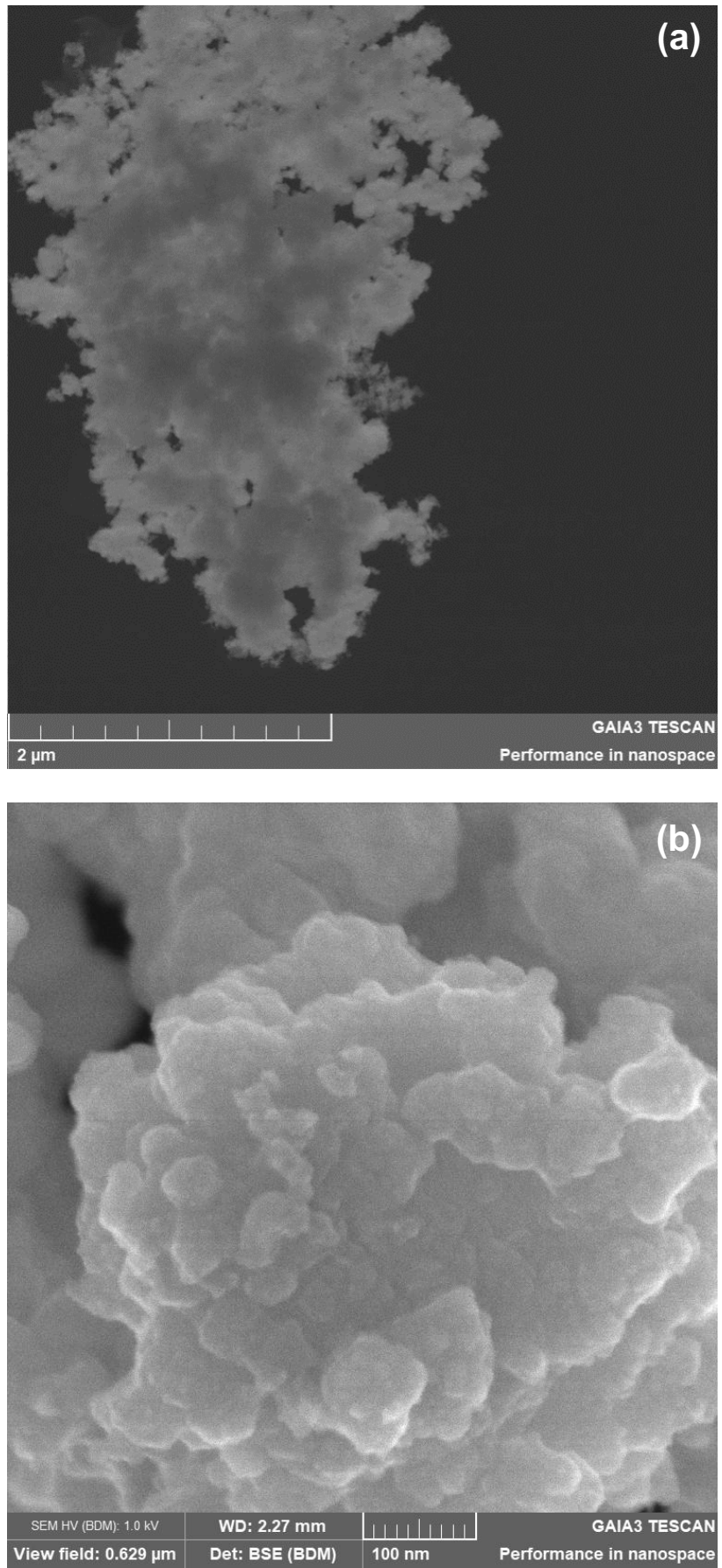

Fig. 2 Secondary-electron (SE) photomicrographs of the investigated sample at variable magnifications. ric triangular velocity shape. The two simultaneously obtained spectra (512 channels each) were folded and evaluated assuming Lorentzian line shape or quadrupole splitting distributions (QSDs) using the program Recoil (Lagarec and Rancourt 1998). During the measurements, the source $\left({ }^{57} \mathrm{Co} / \mathrm{Rh} / 50 \mathrm{mCi}\right)$ and the absorber were kept at room temperature. Two different spectra with different velocity ranges were taken with high count rates of the background of about 4 million counts per channel. Isomer shift (IS) values are reported relative to $\alpha$-iron at room temperature.

\section{Results}

\subsection{Particle morphology, phase and chemical composition}

Figure 2a highlights the morphology of the particles of the investigated products, analysed without further manipulations after centrifugation and washing. The whole characterization was performed on the best available synthetic products. The products were selected, after a preliminary PXRD and SEM/EDS survey, on the basis of:

- the purity of the products (absence of traces of unreacted reactants),

- the qualitative chemical composition (namely the Fe content identifiable in a fast EDS survey),

- the identification of particles with largely different morphology (again, attributable to traces of unreacted products, eventually below the detection limit of the PXRD survey).

Particles consist of aggregates, having anhedral morphology, with the almost complete absence of identifiable crystalline surfaces. Further micrographs revealed the occurrence of individuals up to several tens of $\mu \mathrm{m}$ in size, but the average linear dimension of the particles did not exceed 1-2 $\mu \mathrm{m}$. High-magnification micrographs (Fig. 2b) reveal particles exhibiting complex and rough surfaces, where features of $\sim 10-20 \mathrm{~nm}$ in size are apparent. This evidence suggests that the synthesized particles could have resulted from the aggregation of smaller individuals (of nanometric dimensions, see e.g. Di Benedetto et al. 2011, 2016; Giaccherini et al. 2019b).

The EDX microanalytical determination was performed by both point and raster analyses on the synthetic powders (Tab. 1). The main evidence of the microanalytical results consists in the Fe content. The experimental value is slightly more than a half of the expected one. Conversely, the $\mathrm{Cu}$ content and even more the Sn content, slightly increase over the range 
Fig. 3 Powder X-ray pattern of the investigated sample (in black). The positions of the Bragg reflections are indicated (red markers), as are the indexes of the main reflections for space group $I \overline{4} 2 \mathrm{~m}$.

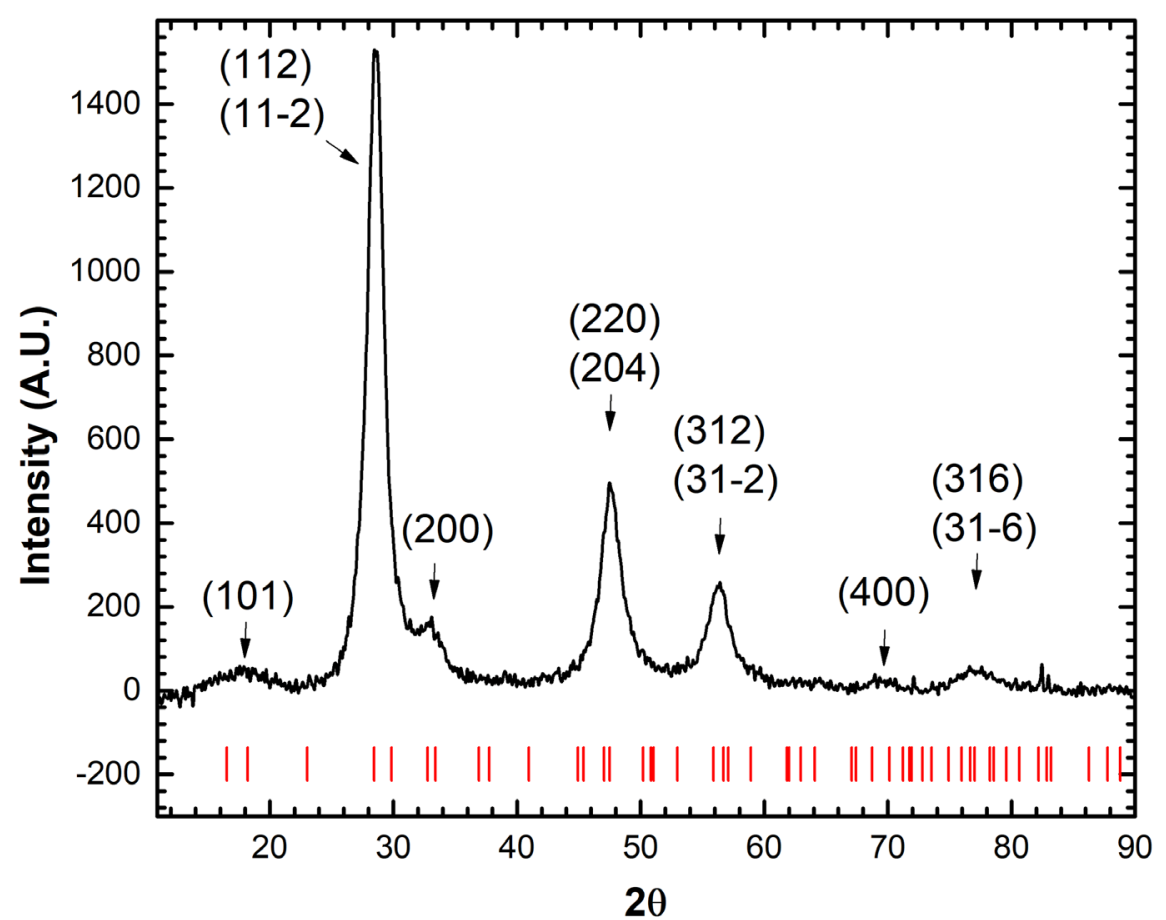

The first signal, occurring at $298 \mathrm{mT}$, is characterised by an asymmetric shape and a width of $\sim 26 \mathrm{mT}$. The $g$ value of the I signal, 2.27 , is perfectly in line with $\mathrm{Cu}(\mathrm{II})$ in a sulfide environment (Peisach and Blumberg 1974). This attribution could explain also the asymmetry and width, which could be due to the occurrence of $\mathrm{Cu}$ (II) in a distorted tetrahedral site (as expected in stannite). In this interpretation, the hyperfine structure expected for a with the COD card 9004745, corresponding to a defe stannite structure crystallizing in the $I \overline{4} 2 \mathrm{~m}$ symmetry. The large width of the reflections (e.g. $1.75^{\circ}$ of $2 \theta$ for 112 ) confirms the small size of the crystallites. An estimate provided by the Rietveld analysis (see Discussion) point to an average size of 14(1) $\mathrm{nm}$.

\subsection{Electron Paramagne- tic Resonance}

The EPR spectrum of the investigated sample reveals the presence of two superimposed signals (labelled as "I" and "II" in the Fig. 4). The two signals differ in position, width and intensity, being clearly attributable to different species.

Fig. 4 Room temperature X-band EPR spectrum of the investigated sample.

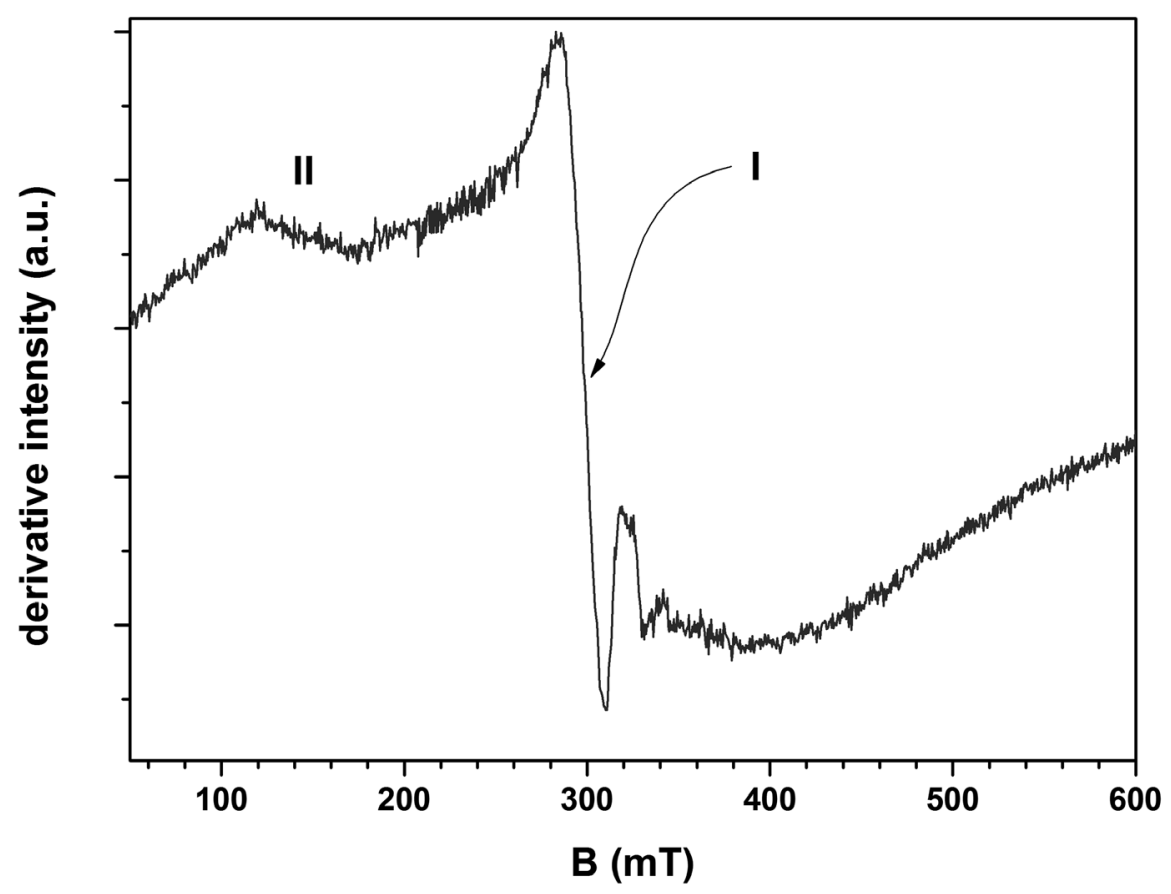



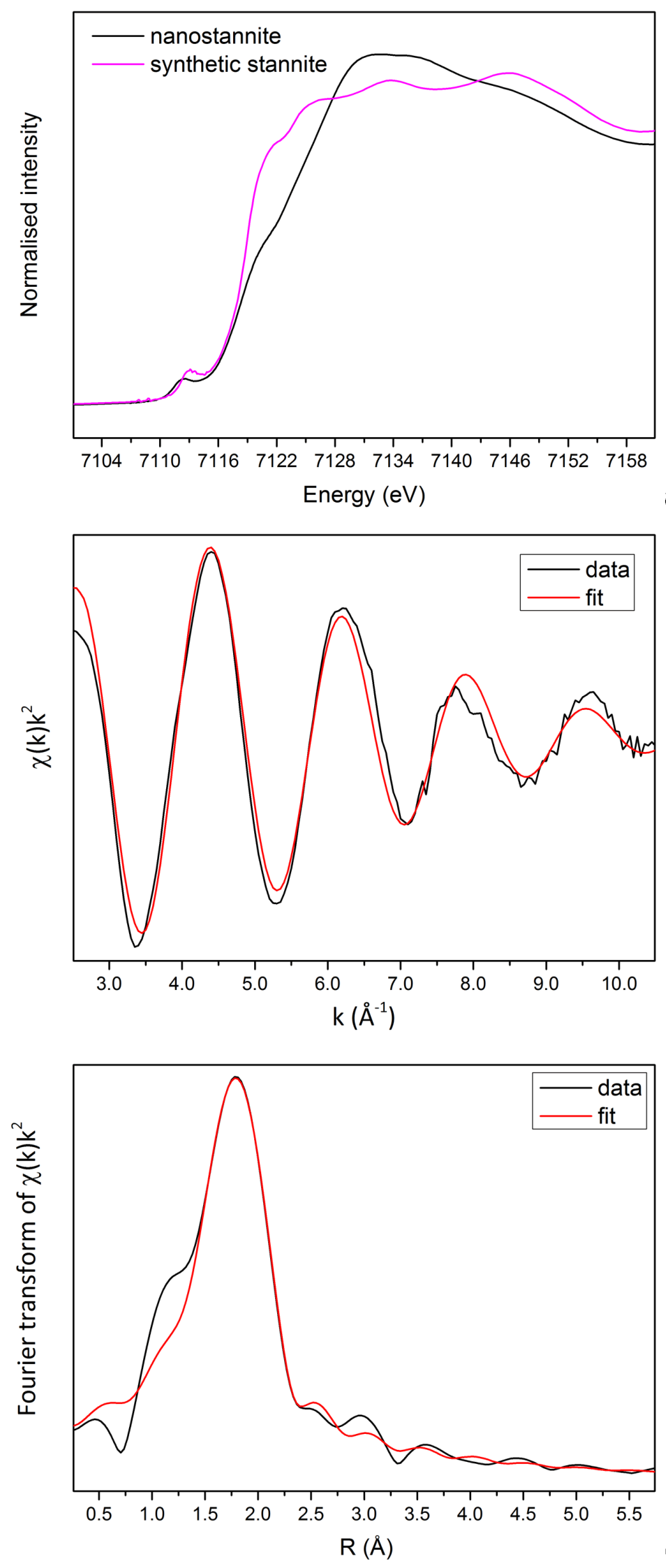

$\mathrm{I}=3 / 2 \mathrm{~S}=1 / 2 \mathrm{~d}^{9} \mathrm{Cu}(\mathrm{II})$ ion would be hidden in the broad width of the signal. Although EPR is not a quantitative technique, the overall amount of $\mathrm{Cu}$ (II) appears in the order of traces, accounting for the signalto-noise ratio (Fig. 4). This is perfectly in line with the general trend of $\mathrm{Cu}$ to occur in its reduced state in sulfide minerals (Makovicky et al. 2006). Several exceptions were reported in the studies of Bente (1987) and Bernardini et al. (2000), and they are often associated to Fe-bearing minerals, so that a partial charge transfer can occur. Anyway, $\mathrm{Cu}(\mathrm{II})$ is always reported to occur as a trace to minor species. Very similar features were observed in other studies concerned with members of the stannite-kesterite pseudobinary system (Bernardini et al. 2000) and other Fe-bearing and Fe-free sulfides (Bente 1987).

The second signal is centered at $\sim 252.5$ $\mathrm{mT}$, with a width of $\sim 276 \mathrm{mT}$. The corresponding $g$ value, $\sim 2.65$, and even more the high width value, support an attribution to $\mathrm{Fe}(\mathrm{III})$. Among the redox states compatible with a sulfide environment, only Fe(III) can be detected by conventional roomtemperature EPR spectroscopy (Goodman and Raynor 1970). The features of the signal shape and position suggest both $\mathrm{Cu}(\mathrm{II})$ and $\mathrm{Fe}$ (III) to occur in a magnetically concentrated environment, i.e. in sites not far from themselves or from the other paramagnetic species, like e.g. Fe(II), present in the sample.

\subsection{X-ray Absorption Spectroscopy}

Figure 5a shows the XAS spectrum in the XANES region of the investigated sample, together with that of synthetic stannite (Bernardini et al. 1990), obtained by hightemperature dry solid-state reactions using a silica glass-tube technique. The absorption edge of the investigated sample occurs at higher energy compared to synthetic stannite and it shows a weak peak, followed by a shoulder on rising absorption. In contrast, synthetic stannite shows a single absorp-

Fig. 5 Normalized XANES spectra of the investigated sample and a synthetic stannite reference compound (a), EXAFS (b) and Fourier transform (c) of the investigated sample. Black lines are the measured data; red lines are fits. 
tion peak, suggesting a mixed state for $\mathrm{Fe}$ in the investigated sample. The edge jump intensity was used to estimate the total amount of $\mathrm{Fe}$ in the sample by comparing its value with several standards with known edge jump and Fe content; it yielded $\approx 0.5$ apfu of Fe.

Figures $5 \mathrm{~b}-\mathrm{c}$ show the EXAFS spectrum, together with its correspondent Fourier transform (FT), for the investigated sample. The FT shows a bimodal I shell peak, while no signal of higher coordination shells is visible. The absence of a second coordination shell is understandable, given the small size of crystallites (e.g. Giaccherini et al. 2019b). Attempts to fit the first shell peak using two different paths (a longer $\mathrm{Fe}^{2+}-\mathrm{S}$ and a shorter $\mathrm{Fe}^{3+}-\mathrm{S}$ ) were unsuccessful and the first shell was modelled using a single Fe-S path with degeneracy of 4 . The shoulder on the low-R side of the FT suggests the presence of an oxidized portion of $\mathrm{Fe}$ in the sample. The EXAFS multiparameter fit results are listed in Tab. 2.

\subsection{Mössbauer spectroscopy}

The Mössbauer spectrum of the investigated samples is shown in the Fig. 6. Apparently, the spectrum consists of a structured absorption, where the presence of more than one component can be inferred. The best-fit results point to the occurrence of the three different doublets, whose isomer shift (IS) and quadrupole splitting (QS) values are listed in the Tab. 3. The three doublets, on the basis of their IS values, are assigned to both Fe(II) (Doublet A) and $\mathrm{Fe}$ (III) (Doublets B and C). These values agree with those already given in the literature (Eibschütz et al. 1967; Vaughan and Burns 1972; Yamanaka and Kato 1976;

Di Benedetto et al. 2005, 2016) for the same valence states in minerals.

The occurrence of significant values of QS confirms the noncubic distribution of the electric field surrounding the Fe ions (in both valence states). Comparing the IS and QS values here presented with those available in the literature (Eibschütz et al. 1967; Vaughan and Burns 1972; Yamanaka and Kato 1976; Di Benedetto et al. 2005, 2016),

Fig. 6 Room-temperature Mössbauer spectrum of the investigated sample and of reference compounds.

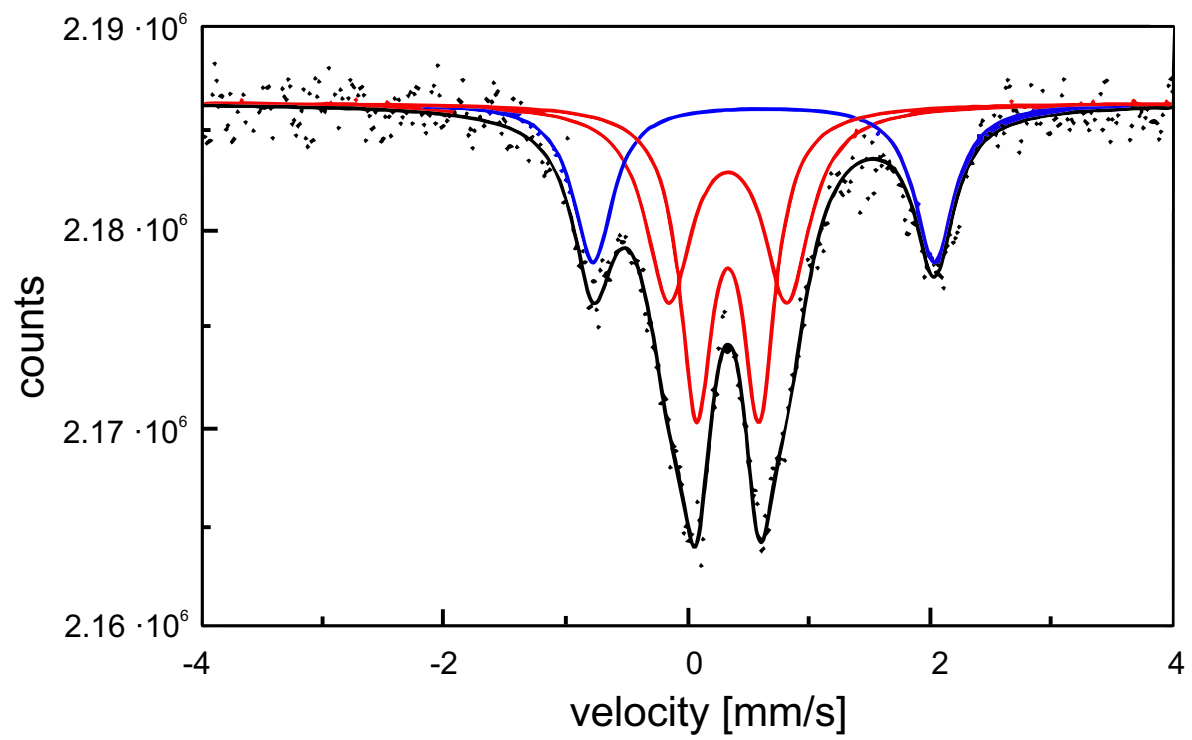

one can assign the $\mathrm{Fe}$ (II) A doublet to $\mathrm{Fe}$ ions in the $2 a$ site. More difficult is the attribution of the $\mathrm{B}$ and $\mathrm{C}$ doublets. The close IS shift values support a very similar attribution for the two doublets. However, the difference in QS, especially taking into account that $\mathrm{Fe}$ (III) is an half-filled $3 \mathrm{~d}$ shell ion, is rather high and definitely points to different attributions. Considering the previous results of Mössbauer spectroscopic studies on $\mathrm{Fe}$ (III) in natural stannite (Di Benedetto et al. 2005; Rusakov et al. 2010), the C doublet can be easily attributed to Fe(III) in the stannite-kuramite framework of the synthesized phase. Conversely, one can tentatively attribute the B doublet to an associated phase, likely a nanosized (thus non-magnetic) oxidized phase. This attribution would be in agreement with the interpretation of the XAS results, which pointed out the occurrence of a Fe(III)-oxide fraction. Thus, Mössbauer spectroscopy depicts a very complex Fe speciation in the samples, where two valence states and at least two different phases are involved.

\subsection{SQUID magnetometry}

The magnetic behavior of the sample as a function of the temperature is shown in Fig. 7. The increase of the $\chi \mathrm{T}$ with increasing temperature closely resembles the correlation recorded for similar metal sulfides (Spalek et al. 1986; Furdyna 1988; Twardowski 1990; Twardowski et al. 1993; Bernardini et al. 2000). A further very common feature of these materials is also the fact that the $\chi \mathrm{T}$ 
Tab. 3 Spectral parameters of the fit refinement of the data of Fig. 6

\begin{tabular}{lcccc}
\hline Doublet & Attribution & IS (mm/s) & QS (mm/s) & Area (\%) \\
\hline A & Fe(II) & $0.627(15)$ & $2.81(3)$ & $25(3)$ \\
B & Fe(III) & $0.332(14)$ & $0.97(12)$ & $36(3)$ \\
C & Fe(III) & $0.331(8)$ & $0.518(43)$ & $39(3)$ \\
Di Benedetto et al. (2016) & $\mathrm{Fe}(\mathrm{III})$ & $0.370(2)$ & $0.648(1)$ & 100 \\
Di Benedetto et al. (2005) & $\mathrm{Fe}(\mathrm{II})$ & 0.61 & 2.89 & 83.3 \\
Di Benedetto et al. (2005) & $\mathrm{Fe}(\mathrm{III})$ & 0.30 & 0.53 & 16.7 \\
Rusakov et al. (2010) & $\mathrm{Fe}(\mathrm{II})$ & 0.62 & 2.9 & 90 \\
Rusakov et al. (2010) & $\mathrm{Fe}(\mathrm{III})$ & 0.33 & 0.5 & 10 \\
\hline
\end{tabular}

data tend to an asymptotic constant value only at a very high temperature (Carlin 1986). This behavior is due to the occurrence, within the sample, of relatively strong magnetic interactions among neighboring magnetic ions that are well described (in the high-temperature regime) by the Curie-Weiss law. In the inset, the results of a fit, carried out between 100 and $298 \mathrm{~K}$, illustrate the good linear trend exhibited by the data, and allow estimating the Curie and Weiss constants at 1.54(2) emu $\mathrm{K} \cdot \mathrm{mol}^{-1}$ and $-48(4) \mathrm{K}$. The Curie constant value implies the fact that the molar fraction of the paramagnetic ions is below that expected for a pure stannite compound (having a nominal spin-only Curie value of $\left.3.0 \mathrm{emu} \cdot \mathrm{K} \cdot \mathrm{mol}^{-1}\right)$. This result is thus in a rough agreement with the chemical composition provided by the SEM-EDX investigation. Concerning the Weiss constant, the experimental value for this sample is in a good agreement with that found for the natural and synthetic stannites $(-32.8$ and $-63.9 \mathrm{~K}$, respectively; Bernardini et al. 2000), especially accounting for the difference in the $\mathrm{Fe}$ content. The nature of interaction, deduced from the sign of the Weiss constant, is antifer-

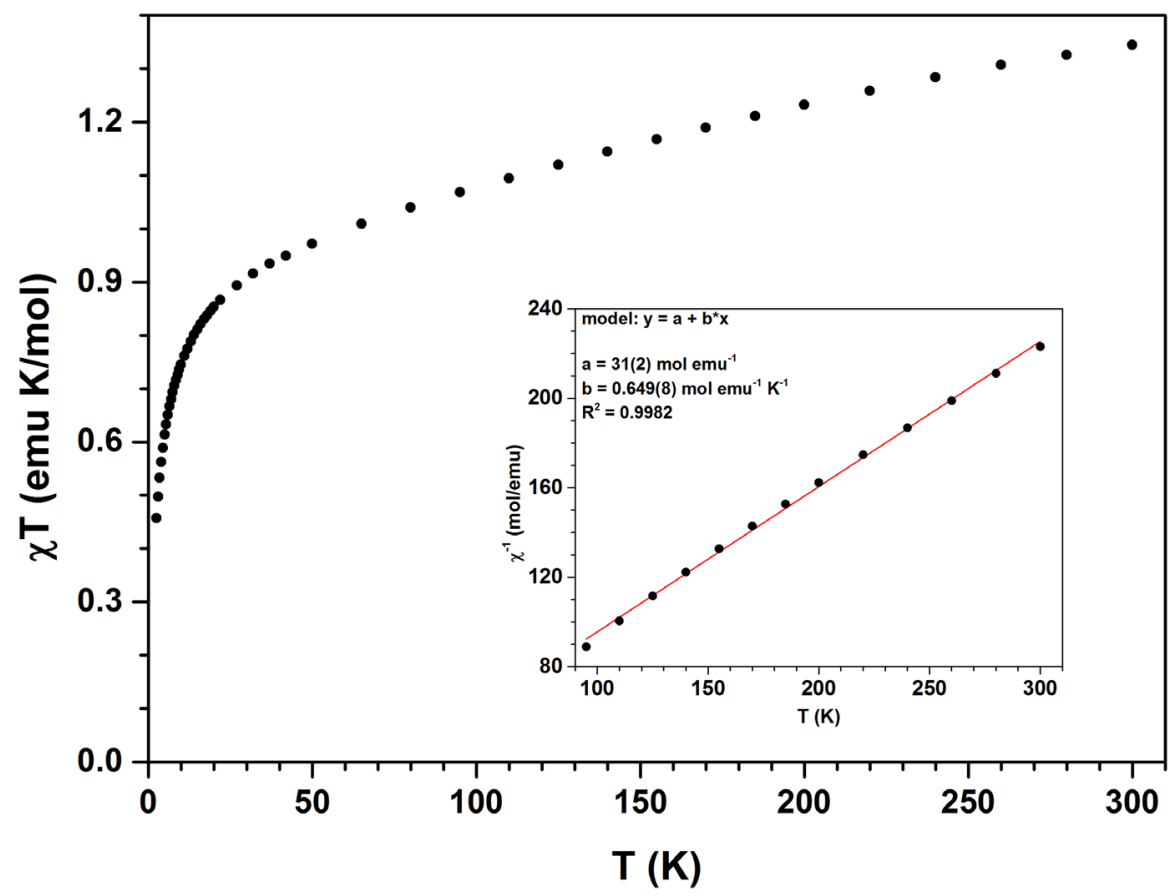

romagnetic. Such a rather high value, due to an appreciable superexchange interaction, clearly suggests that paramagnetic ions (not necessarily $\mathrm{Fe}$ ions, see the Discussion) occur at relatively short distances.

Owing to the fact that for the synthetic stannite the occurrence of antiferromagnetic transition is reported at 6.1(2) K Néel temperature (Caneschi et al. 2004), a closer inspection of the low-temperature regime was performed using a ZFC/FC approach (Fig. 8). A slight but noticeable difference between the ZFC and FC datasets is apparent for T values below $\sim 6 \mathrm{~K}$. A more precise determination for the temperature value of the onset of the irreversible behavior can be provided by the study of the difference between the two datasets $\chi \mathrm{T}_{\mathrm{zfc}}-\chi \mathrm{T}_{\mathrm{fc}}$. When this value exceeds the average uncertainty of the intermediate $\mathrm{T}$ regime (6-40 $\mathrm{K})$, it can be considered as significantly different from the conventional trend. Accordingly, the onset of the irreversible behavior can be set at 4.0(2) K. The nature of this anomalous behavior is in close relation with the findings reported for stannite and related phases (Fries et al. 1997; Caneschi et al. 2004). In these compounds, the presence of antiferromagnetic (AF) interactions in the hightemperature regime is often linked to the occurrence of an $\mathrm{AF}$ transition at low temperature. In the present case, the antiferromagnetic interactions in the high-temperature regime are of the same order of magnitude as those reported in the literature. Thus is conceivable a transition towards a permanent magnetic state at low temperature. However, taking into account the occurrence of just a fraction of the molar content of $\mathrm{Fe}$, and its distribution in the available sites (where at least two sites are randomly occupied), the transition towards an ordered arrangement of magnetic moments, as in an AF state, is unlikely. Thus, the observed transition drives the magnetic behavior of the samples towards a disordered state, such as a spin glass (Carlin 1986; Furdyna 1988).

Fig. $7 \chi \mathrm{T}$ versus $\mathrm{T}$ plot of the investigated sample between 2 and $298 \mathrm{~K}$. Inset: plot of the high-temperature linear regime of the $\chi^{-1}$ versus $\mathrm{T}$ data, modelled with a linear fit (red line). 
Fig. 8 Upper panel: $\chi \mathrm{T}$ versus $\mathrm{T}$ plot of the investigated sample between 2 and $8 \mathrm{~K}$; full black and red circles represent the ZFC and FC datasets, respectively. Lower panel: the differences (with the relative uncertainty $\pm 1 \sigma$ bars) between the two datasets are shown. The light grey region represents the average uncertainty of the difference in the intermediate temperature regime $(6<\mathrm{T}<40 \mathrm{~K})$.

\section{Discussion}

The studied sample is not strictly monophasic, but the associated phase (namely, a Fe oxide) is not revealed by PXRD. We attribute this fact to the occurrence of the Fe oxide association, in the form of poorly crystalline alteration rims of the main sulfide phase. Only a multianalytical spectroscopic investigation was able to detect the presence of such a small fraction of associated phase, practically undetectable by the PXRD alone. The Fe(III) fraction associated to this phase, $36 \%$ of the total Fe according to Mössbauer spectroscopy, can be correlated to an amount of the Fe-oxide in the range of $\sim 4$ wt. \%. Recalling the nanosized dimension of the final products of the synthesis, we suggest that such a small amount of Fe-oxide falls below the detection limit of the PXRD.

Secondly, besides the occurrence of an associated phase, the speciation of metals in the sample, and the related charge balance, is complex. Accounting for $\mathrm{Fe}$ pertaining to the associated phase, and also deriving the occurrence of the Fe(II) and Fe(III) doublets and their relative abundances from the attribution of the Mössbauer spectra, the following general formula (based on $4 \mathrm{~S} \mathrm{pfu}$ ) can be obtained: $\mathrm{Cu}(\mathrm{I})_{2.10} \mathrm{Fe}(\mathrm{II})_{0.16} \mathrm{Fe}(\mathrm{III})_{0.24} \square_{0.38} \mathrm{Sn}_{1.12} \mathrm{~S}_{4.00}$.

As can be seen from the formula, the charge neutrality is mainly reached through a overstoichiometric content of the four-valent $\mathrm{Sn}$ ion and the presence of $\mathrm{Fe}$ (II) and $\mathrm{Fe}(\mathrm{III})$. The small difference among positive and negative charges falls within the experimental uncertainty of the EDX analysis. We did not force part of $\mathrm{Cu}$ to occur as $\mathrm{Cu}(\mathrm{II})$, in line with the fact that the amount of the $\mathrm{Cu}$ (II) revealed by EPR is in the order of traces. This is in agreement with most of the studies on natural stannites and kesterites (Bernardini et al. 1990, 2000; Bonazzi et al. 2003; Rusakov et al. 2010). The upper estimate of the $\mathrm{Cu}$ (II) content can be set to 0.1 apfu. The formula accounts for a small, but relevant, metal deficiency $[\Sigma(\mathrm{Cu}$ $+\mathrm{Fe}+\mathrm{Sn})<4 \mathrm{apfu}$ ], so that cation vacancies are present in the structure. Concerning the anion vacancies, their presence was inferred in the literature especially in relation to low analytical wt. \% totals from EPMA data (see e.g. Bernardini et al. 1990). However, these supposed S-poor contents were always attributed to the difficulties in the calibration of the sulfide minerals. Accordingly, one could consider the presence of anion vacancies to occur, if at all, in a very subordinate amount. The simultaneous presence of cation vacancies and of Fe deficiency coupled to a Sn excess suggests the occurrence of at least partial random site occupancy.

Concerning magnetism, if one considers the content of the two paramagnetic species active in the replacement mechanism, Fe(II) and Fe(III), a molar value of $1.6(2)$ emu $\mathrm{K} / \mathrm{mol}$ is obtained. This calculation assumes the theoretical spin only Curie constants, 3.00 and 4.375 emu $\mathrm{K} / \mathrm{mol}$, for $\mathrm{Fe}(\mathrm{II})$ and $\mathrm{Fe}(\mathrm{III})$, respectively. However, accounting also for the presence of a further associated phase, the efficient Curie constant of the mixture decreases to $1.5(2)$ emu $\mathrm{K} / \mathrm{mol}$, compared to the experimental one $(1.54 \mathrm{emu} \mathrm{K} / \mathrm{mol})$. The associated phase, presumably an Fe oxide and/or hydroxide, is supposed to occur subjected to strong antiferromagnetic interactions or even in an AF state, thus poorly contributing to the overall susceptibility of the sample.

The complete disordering of the cationic sites $(4 d, 2 a$

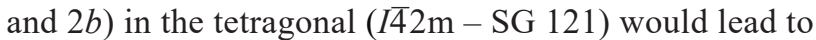
a symmetry increase to $F \overline{4} 3 \mathrm{~m}$ (SG 216) (Di Benedetto et al. 2011; Giaccherini et al. 2019b). The overall effect of the random occupancy in cationic sites is the reduction of the cell volume from the tetragonal cell to a cubic (sphalerite-like) single cell where the lattice constant 
Tab. 4 Results of the Rietveld refinement for cubic $(F \overline{4} 3 \mathrm{~m})$ and tetragonal $(\overline{4} 2 \mathrm{~m})$ models

\begin{tabular}{lccc}
\hline Space group & Unit-cell parameter & Refined value & Bonazzi et al. (2003) \\
\hline \multirow{4}{*}{$I \overline{4} 2 \mathrm{~m}$} & $a$ & $5.445(2)$ & $5.4495(6)$ \\
& $c$ & $10.764(5)$ & $10.726(2)$ \\
& $R_{\mathrm{wp}}$ & $2.35 \%$ & \\
\hline \multirow{4}{*}{$\overline{4} 3 \mathrm{~m}$} & $R_{\mathrm{p}}$ & $1.88 \%$ & $5.406(1)^{\mathrm{a}}$ \\
& $a$ & $5.4113(7)$ & \\
& $R_{\mathrm{wp}}$ & $2.63 \%$ & \\
\hline
\end{tabular}

${ }^{a}$ recalculated from the refined tetragonal lattice parameter values by Bonazzi et al. (2003) with special reference to those necessary for being considered in solar cell applications.

\section{Conclusions}

From the study here presented, the proposed two-step synthesis provides only a partial control of the final phase composition, and

of the cubic cell is related to the tetragonal by $a_{\text {cubic }}=$ $\left(2 a_{\text {tetragonal }}+c_{\text {tetragonal }}\right) / 4$. Figure S1 (in Supporting Information) confirms that the Rietveld refinement of this second structural model exhibits worse agreement factors $\left(R_{\mathrm{wp}}\right.$ $2.35 \%$ and $2.63 \%$ for tetragonal and cubic models, respectively) with the experimental data (Tab. 4). No preferential orientation is necessary to fit the models and the unit cells in the $I \overline{4} 2 \mathrm{~m}$ and $F \overline{4} 3 \mathrm{~m}$ space groups compare well with unit-cell parameters reported by Bonazzi et al. (2003) (Tab. 4). The better fit at low $2 \theta$ angle values (below $24^{\circ} 2 \theta$ ) favors the tetragonal structural model. Hence, the products crystallize in the $I \overline{4} 2 \mathrm{~m}$ symmetry with an undetermined (partly random) distribution of cations, at least in the $2 a$ cationic site.

The present results allow highlighting some general features of the investigated synthetic stannite:

1) prepared experimental product shows significant deficit of $\mathrm{Fe}(0.40 \mathrm{pfu})$ and the $\mathrm{Fe}$ stoichiometry does not correspond to the molar ratio of the reactants,

2) the charge balance is achieved by presence of $\mathrm{Fe}(\mathrm{III})$ and over stoichiometry of Sn(IV),

3) the site symmetry of the site(s) occupied by $\mathrm{Cu}$ (II) and Fe species is lower than cubic.

All the above considerations reveal a crystal chemical behavior of these solvothermal materials significantly more complicated than the situation observed for natural stannite-related minerals (e.g. Hall et al. 1978; Bernardini et al. 1990, 2000; Bonazzi et al. 2003; Schorr et al. 2007). As stated also for other solvothermal compounds (Di Benedetto et al. 2011; Giaccherini et al. 2019a), one can consider the solvothermal products eminently different from the natural ones, in terms of metal distribution among the available sites. Besides the obvious difference in the reaction environment, one can also attribute this difference to the cooling of the sample from the reaction temperature (Schorr et al. 2007): here the powders were let to cool down spontaneously at room temperature. The question of the metal distribution in the available cationic sites is particularly relevant, if these materials are considered for the design and development of new solar cell devices. Schorr (2011), in fact, has pointed out the importance of antisite defects (and vacancies) in determining the physical properties of the resulting product, of the crystal structure of the multinary sulfide. The same holds true also as far as the overall chemical composition is concerned. The difference between the stoichiometric and the observed composition of the products clearly marks the system thermodynamics (i.e. driven by the different solubility products of the binary metal-sulfur compounds in the ethylene glycol - EG) as the main actor involved in the determination of the final composition. Moreover, we attribute a relevant role also to the redox state of $\mathrm{Fe}$. In principle, the general environment of the chosen synthesis should be considered as reducing given the reducing potential of thiourea; clearly, the EG itself and the air exposure of the reaction vessel (i.e. some $\mathrm{O}_{2}$ from air could be dissolved in EG) can be considered oxidizing agents, and they can affect the reagents. This could trigger the production of $\mathrm{Fe}$ oxide, limiting the sulfide uptake, and allowing the partial oxidation of Fe(II) to $\mathrm{Fe}(\mathrm{III})$. As a result, we observe that $\mathrm{Fe}$ (III) sums up to $3 / 4$ of the total $\mathrm{Fe}$. The presence of such a large number of $\mathrm{Fe}(\mathrm{III})$ ions in the structure can be accommodated only by inserting a certain number of vacancies. The choice of avoiding any unnecessary chemical has resulted in the incapability of setting and buffering the redox potential during the synthesis.

The present study allows deep insight into the crystal chemistry of the solvothermal multinary sulfides, revealing an astonishingly complicate situation, and to gain a better understanding of the laws that control the final properties of the products, especially those of interest in solar cell applications.

Acknowledgements. The authors gratefully acknowledge František Laufek and an anonymous reviewer for their critical reading of the manuscript. Authors are also indebted to E. Berretti for his help during the FEG/SEM investigations. This study was realized under the financial and logistic support by Università di Firenze (Progetto di Ateneo funds to FDB), Italian CNR (CEME), Ente Cassa di Risparmio di Firenze (to AL). X-ray Absorption Spectroscopy (XAS) measurements were carried out at European Synchrotron Radiation Facility (ESRF), which is gratefully acknowledged for provision of the synchrotron radiation and access to the facility. 
Electronic supplementary material. Supplementary material concerning XRPD Rietveld interpretation in different Space Groups is available online at the Journal web site (http://dx.doi.org/10.3190/jgeosci.300).

\section{References}

Alanazi AM, Alam F, Salhi A, Missous M, Thomas AG, O'BRIEN P, LEWIS DJ (2019) A molecular precursor route to quaternary chalcogenide $\mathrm{CFTS}\left(\mathrm{Cu}_{2} \mathrm{FeSnS}_{4}\right)$ powders as potential solar absorber materials. Rsc Adv 9: 24146-24153

Alsema E (2012) Energy payback time and $\mathrm{CO}_{2}$ emissions of PV systems. In: McEvoy A, MARKvart T, CASTAÑER L (eds) Practical Handbook of Photovoltaics, $2^{\text {nd }}$ Edition. Fundamentals and Applications. Academic Press, Elsevier, Amsterdam, pp 1097-1117

Ankudinov AL, Ravel B, ReHr JJ, ConRadson SD (1998) Real-space multiple-scattering calculation and interpretation of X-ray-absorption near-edge structure. Phys Rev B 58: 7565-7576

BAIN GA, BERry JF (2008) Diamagnetic corrections and Pascal's constants. J Chem Educ 85: 532-536

BENTE K (1987) Stabilization of Cu-Fe-Bi-Pb-Sn-sulfides. Mineral Petrol 36: 205-217

Bernardini GP, Bonazzi P, Corazza M, Corsini F, Mazzetti G, Poggi L, Tanelli G (1990) New data on the $\mathrm{Cu}_{2} \mathrm{FeSnS}_{4}-\mathrm{Cu}_{2} \mathrm{ZnSnS}_{4}$ pseudobinary system at $750^{\circ}$ and $550^{\circ} \mathrm{C}$. Eur J Mineral 2: 219-225

Bernardini GP, Borrini D, CANESChi A, Di Benedetto F, Gatteschi D, Ristori S, Romanelli M (2000) EPR and SQUID magnetometry study of $\mathrm{Cu}_{2} \mathrm{FeSnS}_{4}$ (stannite) and $\mathrm{Cu}_{2} \mathrm{ZnSnS}_{4}$ (kesterite). Phys Chem Miner 27: 453-461

Bonazzi P, Bindi L, Bernardini GP, Menchetti S (2003) A model for the mechanism of incorporation of $\mathrm{Cu}, \mathrm{Fe}$ and $\mathrm{Zn}$ in the stannite-kesterite series, $\mathrm{Cu}_{2} \mathrm{FeSnS}_{4}-$ $\mathrm{Cu}_{2} \mathrm{ZnSnS}_{4}$. Canad Mineral 41: 639-647

Caneschi A, Cipriani C, Di Benedetto F, Sessoli R (2004) Characterisation of the antiferromagnetic transition of $\mathrm{Cu}_{2} \mathrm{FeSnS}_{4}$, the synthetic analogue of stannite. Phys Chem Miner 31: 190-193

CARLin RL (1986) Magnetochemistry. Springer, Berlin, Heidelberg, New York, pp 1-368

Cui Y, Deng R, Wang G, PAn D (2012) A general strategy for synthesis of quaternary semiconductor $\mathrm{Cu}_{2} \mathrm{MSnS}_{4}$ $\left(\mathrm{M}=\mathrm{Co}^{2+}, \mathrm{Fe}^{2+}, \mathrm{Ni}^{2+}, \mathrm{Mn}^{2+}\right)$ nanocrystals. J Mater Chem 22: 23136-23140

D'Acapito F, Lepore GO, Puri A, Laloni A, La Manna F, Dettona E, De Luisa A, Martin A (2019) The LISA beamline at ESRF. J Synchrotron Radiat 26: 551-558

Di Benedetto F, Bernardini GP, Borrini D, LotTerMoser W, Tippelt G, Amthauer G (2005) ${ }^{57} \mathrm{Fe}$ - and ${ }^{119} \mathrm{Sn}$-Mössbauer study on stannite $\left(\mathrm{Cu}_{2} \mathrm{FeSnS}_{4}\right)$-kesterite $\left(\mathrm{Cu}_{2} \mathrm{ZnSnS}_{4}\right)$ solid solution. Phys Chem Miner 31: 683-690
Di Benedetto F, Borrini D, Caneschi A, Fornaciai G, InNocenti M, LaVacchi A, Massa CA, Montegrossi G, Oberhauser W, Pardi LA, Romanelli M (2011) Magnetic properties and cation ordering of nanopowders of the synthetic analogue of kuramite, $\mathrm{Cu}_{3} \mathrm{SnS}_{4}$. Phys Chem Miner 38: 483-490

Di Benedetto F, Bencistà I, D’Acapito F, Frizzera S, CaNeschi A, InNOCEnti M, LaVACChi A, Montegrossi G, Oberhauser W, Romanelli M, Dittrich H, Pardi LA, Tippelt G, Amthauer G (2016) Geomaterials related to photovoltaics: a nanostructured Fe-bearing kuramite, $\mathrm{Cu}_{3} \mathrm{SnS}_{4}$. Phys Chem Miner 43: 535-544

Eibschütz M, Hermon E, Shtrikman S (1967) Determination of cation valencies in $\mathrm{Cu}_{2}^{57} \mathrm{Fe}^{119} \mathrm{SnS}_{4}$ by Mössbauer effect and magnetic susceptibility measurements. J Phys Chem Solids 28: 1633-1636

Fries T, Shapira Y, Palacio F, Morón MC, McIntyre GJ, Kershaw R, Wold A, McNiff JR EJ (1997) Magnetic ordering of the antiferromagnet $\mathrm{Cu}_{2} \mathrm{MnSnS}_{4}$ from magnetisation and neutron scattering measurements. Phys Rev (B) 56: 5424-5431

FURDYNA JK (1988): Diluted magnetic semiconductors. J Appl Phys 64: R29-R64

Giaccherini A, Cucinotta G, Martinuzzi S, Berretti E, Oberhauser W, LaVacchi A, Lepore GO, Montegrossi G, Romanelli M, De Luca A, Innocenti M, Moggi Cecchi V, Mannini M, Buccianti A, Di Benedetto F (2019a) Green and scalable synthesis of nanocrystalline kuramite. Beilstein J Nanotechnol 10: 2073-2083

Giaccherini A, Baldassarre A, Donini L, Lepore GO, Caneschi A, De Luca A, Innocenti M, Montegrossi G, Cucinotta G, Oberhauser W, Pardi L, Romanelli M, Mannini M, Di Benedetto F (2019b) Sustainable synthesis of quaternary sulphides: the problem of the uptake of zinc in CZTS. J Alloys Compd 775: 1221-1229

Giraldo S, Jehl Z, Placidi M, IzQuierdo-Roca V, PéreZRodríguez A, SAucedo E (2019) Progress and perspectives of thin film kesterite photovoltaic technology: a critical review. Adv Mater 31: 1806692

Goodman BA, RaYNor JB (1970) Electron spin resonance of transition metal complexes. In: EMELÉNS HJ, Sharpe AG (eds) Advances in Inorganic Chemistry and Radiochemistry. Academic Press, New York, London, pp 135-161

Gusain M, Rawat P, Nagarajan R (2015) Facile synthesis and optical properties of pure and $\mathrm{Ni}^{2+}, \mathrm{Co}^{2+}, \mathrm{Bi}^{3+}, \mathrm{Sb}^{3+}$ substituted $\mathrm{Cu}_{3} \mathrm{SnS}_{4}$. RSC Adv 5: 43202-43208

Hall SR, Szymanski JT, Stewart JM (1978) Kesterite, $\mathrm{Cu}_{2}(\mathrm{Zn}, \mathrm{Fe}) \mathrm{SnS}_{4}$, and stannite, $\mathrm{Cu}_{2}(\mathrm{Fe}, \mathrm{Zn}) \mathrm{SnS}_{4}$, structurally similar but distinct minerals. Canad Mineral 16: 131-137

Hou H, Guan H, Li L (2017) Synthesis of $\mathrm{Cu}_{2} \mathrm{FeSnS}_{4}$ thin films with stannite and wurtzite structure directly on glass substrates via the solvothermal method. J Mater Sci-Mater El 28: 7745-7748 
Hussein H, Yazdani A (2019) Doping the bismuth into the host's $\mathrm{Cu}_{2} \mathrm{ZnSnS}_{4}$ semiconductor as a novel material for thin film solar cell. Results Phys 12: 1586-1595

Kovalenker VA (1981) Kuramite, $\mathrm{Cu}_{3} \mathrm{SnS}_{4}$, a new mineral of the stannite group. Int Geol Rev 23: 365-370

LAGAREC K, RANCOURT DG (1998) Extended Voigt-based analytic line shape method for determining $\mathrm{N}$-dimensional correlated hyperfine parameter distributions in Mössbauer spectroscopy. Nucl Instrum Methods 129: 266-280

LeE PA, Citrin PH, Eisenberger P, Kincaid BM (1981) Extended X-ray absorption fine structure - its strengths and limitations as a structural tool. Rev Mod Phys 53: 769-806

MAKovicky E (2006) Crystal structures of sulfides and other chalcogenides. In: VAUGHAN DJ (ed) Sulfide Mineralogy and Geochemistry. Mineralogical Society of America Reviews in Mineralogy 61: 7-126

Mukurala N, Mishra, RK, Jin SH, Kushwaha AK (2019) Sulphur precursor dependent crystallinity and optical properties of solution grown $\mathrm{Cu}_{2} \mathrm{FeSnS}_{4}$ particles. Mater Res Express 6(8) 085099

Nefzi C, Souli M, Cuminal Y, Kamoun-Turki N (2018) Effect of sulfur concentration on structural, optical and electrical properties of $\mathrm{Cu}_{2} \mathrm{FeSnS}_{4}$ thin films for solar cells and photocatalysis applications. Superlattice Microst 124: 17-29

Nilange SG, Patil NM, Yadav AA (2019) Influence of precursor thiourea contents on the properties of spray deposited $\mathrm{Cu}_{2} \mathrm{FeSnS}_{4}$ thin films. Physica B 570: 73-81

Oueslati H, Ben Rabeh M, KanZari M (2018) Growth and characterization of the evaporated quaternary absorber $\mathrm{Cu}_{2} \mathrm{FeSnS}_{4}$ for solar cell applications. J El Mater 47: 3577-3584

Pavel CC, Lacal-Arántegui R, Marmier A, Schüler D, Tzimas E, Buchert M, Jenseit W, Blagoeva D (2017) Substitution strategies for reducing the use of rare earths in wind turbines. Resour Policy 52: 349-357

Peisach J, Blumberg WD (1974) Analysis of EPR copper: structural implications derived from the analysis of EPR spectra of natural and artificial Cu-proteins. Arch Biochem Biophys 165: 691-708

Puri A, LePore GO, D'ACAPITO F (2019) The new beamline LISA at ESRF: performances and perspectives for Earth and environmental sciences. Condens Matter 4: 12

RAVEL B (2001) ATOMS: crystallography for the X-ray absorption spectroscopist. J Synchrotron Radiat 8: 314-316

Ravel B, NewVILle M (2005) ATHENA, ARTEMIS, HEPHAESTUS: data analysis for X-ray absorption spectroscopy using IFEFFIT. J Synchrotron Radiat 12: 537-541

Riha SC, PArkinson BA, Prieto AL (2009) Solution-based synthesis and characterization of $\mathrm{Cu}_{2} \mathrm{ZnSnS}_{4}$ nanocrystals. J Am Chem Soc131: 12054-12055

Rusakov VS, Chistyakova NI, Burkovsky IA, GapochKa AM, Evstigneeva TL, Schorr S (2010) Mössbauer study of isomorphous substitutions in $\mathrm{Cu}_{2} \mathrm{Fe}_{1-\mathrm{x}} \mathrm{Cu}_{\mathrm{x}} \mathrm{SnS}_{4}$ and $\mathrm{Cu}_{2} \mathrm{Fe}_{1-\mathrm{x}} \mathrm{Zn}_{\mathrm{x}} \mathrm{SnS}_{4}$ series. J Phys Conf Series 217: 012038

SANAD MMS, ElsEMAN AM, ELSENETy MM, RASHAD MM, ELSAYED B (2019) Facile synthesis of sulfide-based chalcogenide as hole-transporting materials for cost-effective efficient perovskite solar cells. J Mater Sci-Mater E1 30: 6868-6875

SCHORR S (2011) The crystal structure of kesterite type compounds: a neutron and X-ray diffraction study. Solar Energ Mat Sol C 95:1482-1488

Schorr S, HoEbler H-J, Tovar M (2007) A neutron diffraction study of the stannite-kesterite solid solution series. Eur J Mineral 19: 65-73

SPALEK J, LEWICKI A, TARnAWSKi Z, Furdyna JK, GalaZKa RR, OвUsZко Z (1986) Magnetic susceptibility of semimagnetic semiconductors: the high temperature regime and the role of superexchange. Phys Rev B 33: 3407-3418

ToBy BH, Von DreELE RB (2013) GSAS-II : the genesis of a modern open-source all purpose crystallography software package. J Appl Crystallogr 46: 544-549

Twardowski A (1990) Magnetic properties of Fe-based diluted magnetic semiconductors. J Appl Phys 67: 5108-5113

Twardowski A, Fries T, Shapira Y, Demianiuk M (1993) The $\mathrm{d}-\mathrm{d}$ exchange interaction in the diluted magnetic semiconductor ZnFeS. J Appl Phys 73: 6087-6089

Vaughan DJ, Burns RG (1972) Mössbauer spectroscopy and bonding in sulphide minerals containing four-coordinated iron. In:. Proceedings of the $24^{\text {th }}$ International Geological Congress, 21-30 September 1972, Montreal, pp 158-167

YamanaKa T, KaTo A (1976) Mössbauer effect study of ${ }^{57} \mathrm{Fe}$ and ${ }^{119} \mathrm{Sn}$ in stannite, stannoidite, and mawsonite. Amer Miner 61: 260-265

Zaman MB, Mir RA, Poolla R (2019a) Growth and properties of solvothermally derived highly crystalline $\mathrm{Cu}_{2} \mathrm{ZnSnS}_{4}$ nanoparticles for photocatalytic and electrocatalytic applications. Int J Hyd Energy 44: 23023-23033

Zaman MB, Chandel T, Poolla R (2019b) Hydrothermal synthesis of $\mathrm{Cu}_{2} \mathrm{FeSnS}_{4}$ anisotropic nanoarchitectures: controlled morphology for enhanced photocatalytic performance. Mater Res Express 6: 075058

Zaman MB, Chandel T, Poolla R (2019c) Solvothermal synthesis of $\mathrm{Cu}_{2} \mathrm{SnSe}_{3}$ nanocrystals using elemental precursors: influence of different solvents on growth and morphology. J El Mater 48: 3194-3207

Zhong J, Wang Q, Chen D, Chen L, Yu H, Lu H, Ji Z (2015) Biomolecule-assisted solvothermal synthesis of 3D hierarchical $\mathrm{Cu}_{2} \mathrm{FeSnS}_{4}$ microspheres with enhanced photocatalytic activity. Appl Surf Sci 343: 28-32

ZHOU J, Hu Y, Chen X (2016) Preparation of photovoltaic absorption material $\mathrm{Cu}_{2} \mathrm{FeSnSe}_{4}$ microparticles via an atmospheric pressure liquid reflux method. Mater Lett 184: 216-218 